\title{
On the Question of the Thompson Group
}

\author{
Jaeseong HEO* \\ Department of Mathematics, Hanyang University, Seoul 133-791, Korea
}

Received February 16, 2009; final version accepted June 9, 2009

\begin{abstract}
This survey is concerned with the Thompson group $F$ and the von Neumann algebra $L(F)$ associated with $F$. We first review some basic results on the Thompson groups and group von Neumann algebras. We investigate the structure of the von Neumann algebra $W^{*}(F, P)$ generated by $L(F)$ and a projection $P$ on $l^{2}(F)$. We show that the algebra (not necessarily $*$ ) algebraically generated by two generating unitaries of $L(F)$ and the commutant $L(F)^{\prime}$ is strong-operator dense in $\mathcal{B}(\mathscr{H})$. Furthermore, we will discuss actions of $F$ and $F^{\prime}$ on a set of dyadic rational numbers in $[0,1]$ and some outer automorphism of $L(F)$ for the amenability question of $F$.
\end{abstract}

KEYWORDS: Thompson group, normal form, transitive algebra, amenable action, co-amenability, approximately inner automorphism

\section{Introduction}

The Thompson group $F$ was introduced in the 1960s by Richard J. Thompson in connection with studies in logic. It was used to construct finitely presented groups with unsolvable word problems. The Thompson group is known as a very interesting group and it appears in a variety of mathematical areas: word problems, dynamical systems, homotopy theory, group cohomology and analysis. The Thompson group $F$ can be realized as the group of piecewise linear and orientation-preserving homeomorphisms of $[0,1]$ which, except at finitely many dyadic rational numbers, are differentiable with derivatives equal to powers of 2 . In this survey we are concentrated on the amenability of discrete groups, especially, on the amenability question for the Thompson group $F$.

A discrete group $G$ is called amenable if there is a left translation invariant state on $l^{\infty}(G)$. Von Neumann introduced the amenability of groups and proved that the non-abelian free group $\mathbb{F}_{2}$ with two generators is not amenable. Furthermore, he proved that the class of all amenable groups contains all abelian groups and all finite groups, and is closed under quotients, subgroups, extensions, and directed unions with respect to inclusions. He conjectured that any non-amenable group would contain a copy of $\mathbb{F}_{2}$ as a subgroup. A counterexample was given by Ol'shanskii, that is, using Grigochuck's cogrowth criterion for amenability he proved that there exists a non-amenable group such that every proper subgroup is cyclic. The amenability question of the Thompson group $F$ has been posed by Geoghegan. In 1979, he conjectured that the Thompson group $F$ does not contain a non-abelian free subgroup and that the Thompson group $F$ is non-amenable. It was proved by Brin and Squier [2] that the Thompson group $F$ has no free non-abelian subgroups. However, the non-amenability problem of the Thompson group $F$ is still open. This non-amenability problem is of considerable interest since $F$ is expected to be another counterexample to the von Neumann's conjecture for finitely presented groups.

To prove that the Thompson group $F$ is not amenable, Grigorchuk [6] suggested to show that $H_{b}^{n}(F, \mathbb{R}) \neq 0$ for some positive integer $n$ where $b$ indicates the bounded group cohomology. But we think that it is very difficult to compute higher cohomology groups $H_{b}^{n}(F, \mathbb{R})$. Thus, we would like to suggest another approach for proving the non-amenability of the Thompson group $F$.

This survey is organized as follows: the preliminary section prepares some basic results on the Thompson groups and group von Neumann algebras. We collect some known facts and also folklore-like facts about the Thompson group $F$ and group von Neumann algebras. In the third section we show that $W^{*}\left(F, P_{x}\right)$ is of type II or of type I, where $W^{*}\left(F, P_{x}\right)$ is the von Neumann algebra generated by $L(F)$ and an orthogonal projection $P_{x}$ of $l^{2}(F)$ onto $l^{2}(F(x)$ ) where $F(x)$ is the set of elements in $F$ with their normal forms starting with $x$. It is proved that the algebra algebraically (not necessarily *) generated by two unitaries $\left\{L_{x_{0}}, L_{x_{1}}\right\}$ and the commutant $L(F)^{\prime}$ is strong-operator dense in $\mathscr{B}(\mathcal{H})$. The fourth section contains definitions of an amenable action and co-amenability of groups. It is proved that some subgroup $F_{I}$ of $F$ is coamenable in $F$. Finally, we introduce an order two automorphism $\alpha$ of $L(F)$ which is not inner and ask if $\alpha$ is not approximately inner for non-amenability of the Thompson group $F$. 


\section{Some Properties of $F$ and von Neumann algebras}

Let $G$ be a countable discrete group with the identity $e$ and let $\mathscr{H}$ be the Hilbert space $l^{2}(G)$ with the usual inner product. Let $L(G)$ be the von Neumann algebra generated by $\left\{L_{g}: g \in G\right\}$ where $L_{g}$ denotes the left translation of functions in $\mathscr{H}$ by $g^{-1}$. Similarly, we denote by $R(G)$ the von Neumann algebra generated by $\left\{R_{g}: g \in G\right\}$ where $R_{g}$ is the right translation by $g$ on $\mathcal{H}$. Then we have that $L(G)^{\prime}=R(G)$ and $R(G)^{\prime}=L(G)$ where $\mathcal{M}^{\prime}$ is the commutant of $\mathcal{M}$ in $\mathcal{B}(\mathcal{H})$. In general, $L(G)$ and $R(G)$ are finite von Neumann algebras since they have a faithful normal trace $\tau(x)=\left\langle x\left(\chi_{e}\right), \chi_{e}\right\rangle, x \in L(G)$ (or $x \in R(G)$ ). The algebras $L(G)$ and $R(G)$ are factors (of type $\mathrm{II}_{1}$ ) precisely when each conjugacy class in $G$ (other than that of $e$ ) is infinite. In this case we say that $G$ is an infinite conjugacy class (i.c.c.) group. We refer to [16] for the theory of von Neumann algebras as well as the construction of group von Neuamnn algebras.

Definition 2.1. Let $\mathcal{M}$ be a type $\mathrm{II}_{1}$-factor with with a normalized faithful trace $\tau$.

(i) $\mathcal{M}$ has the property $\Gamma$ if, for each $x_{1}, \ldots, x_{n} \in \mathcal{M}$ and $\epsilon>0$, there exists a unitary $u \in \mathcal{M}$ such that $\tau(u)=0$ and $\left\|u x_{i} u^{*}-x_{i}\right\|_{2}<\epsilon(i=1, \ldots, n)$, where for $x \in \mathcal{M},\|x\|_{2}=\tau\left(x^{*} x\right)^{1 / 2}$.

(ii) $\mathcal{M}$ is a McDuff factor if $\mathcal{M}$ is $*$-isomorphic to the tensor product $\mathcal{M} \otimes \mathcal{R}$ where $\mathcal{R}$ is the hyperfinite $\mathrm{II}_{1}$-factor.

This is a weaker form of amenability since the group von Neumann algebra $L(G)$ is a McDuff factor whenever $G$ is a countable amenable i.c.c. group. However, the converse is not true: as is well known, there exist uncountable families of non-amenable i.c.c. groups such that the associated factors are pairwise non-isomorphic McDuff factors. This means that the Thompson group $F$ still has a good chance of being non-amenable. We would like to remark that McDuff property implies property $\Gamma$ for $\mathrm{II}_{1}$-factors. Following E. Effros [5], a discrete group $G$ is inner amenable if $G$ possesses a mean $m$ which is invariant under inner automorphisms and nontrivial, i.e., $m(\{e\}) \neq 1$. Since any invariant mean on $G$ is a non-trivial inner invariant mean on $G$, the inner amenability is a weaker property than the amenability. Effros [5] observed that if $G$ is an i.c.c. group and if the von Neumann algebra $L(G)$ has property $\Gamma$, then $G$ is inner amenable. However, the converse is still open.

The following property has been introduced by Sakai in the 70's and consists essentially of a stronger requirement than property $\Gamma$ of Murray and von Neumann: instead of a sequence of unitaries almost commuting with the elements of the factor one wants a sequence of (inner) $*$-isomorphisms. Moreover, asymptotic abelianness is a stronger property than the McDuff property. For some examples of asymptotically abelian factors, we refer to [7].

Definition 2.2. A finite factor $\mathcal{M}$ is asymptotically abelian if there exists a sequence of $*$-automorphisms $\left(\phi_{n}\right)_{n \in \mathbb{N}}$ on $\mathcal{M}$ such that $\left\|\left[\phi_{n}(x), y\right]\right\|_{2} \rightarrow 0$ for $x, y \in \mathcal{M}$. If each $\phi_{n}$ is inner then $\mathcal{M}$ is called inner asymptotically abelian.

We now review some properties of the Thompson group $F$. We refer the expository note [3] for a good introduction, more details and historical remarks to $F$. The Thompson group $F$ is the set of piecewise linear homeomorphisms from the closed unit interval $[0,1]$ onto itself that are differentiable except at finitely many dyadic rational numbers and such that on intervals of differentiability the derivatives are powers of 2 . The Thompson group $F$ has the following presentation

$$
F=\left\langle x_{0}, x_{1}, \ldots \mid x_{i}^{-1} x_{n} x_{i}=x_{n+1}, 0 \leq i<n\right\rangle .
$$

From this relation $x_{i}^{-1} x_{n} x_{i}=x_{n+1}(0 \leq i<n)$, we have the relation $x_{n+1}=x_{0}^{-n} x_{1} x_{0}^{n}$ for $n \geq 1$, so that $F$ is generated by $x_{0}$ and $x_{1}$. If $F_{1}$ is a group defined by

$$
F_{1}=\left\langle A, B \mid\left[A B^{-1}, A^{-1} B A\right]=e,\left[A B^{-1}, A^{-2} B A^{2}\right]=e\right\rangle,
$$

then there exists a group isomorphism from $F_{1}$ onto $F$ which maps $A$ to $x_{0}$ and $B$ to $x_{1}$. This is obtained by declaring $x_{0}=A$ and $x_{n}=A^{-(n-1)} B A^{n-1}$. Hence the Thompson group $F$ has the finite presentation with two generators and two relators. The following proposition is very useful when one works on the Thompson group.

Theorem 2.3 ([3]). Let $F$ be the Thompson group.

(1) Let $0=a_{0}<a_{1}<\cdots<a_{n}=1$ and $0=b_{0}<b_{1}<\cdots<b_{n}=1$ be partitions of $[0,1]$ consisting of dyadic rational numbers. Then there is $x \in F$ such that $x\left(a_{i}\right)=b_{i}$ for $i=0,1, \ldots, n$.

(2) Every non-trivial element $x$ of $F$ can be expressed in a unique form

$$
x=x_{i_{1}} \cdots x_{i_{m}} x_{j_{k}}^{-1} \cdots x_{j_{1}}^{-1}
$$

where $0 \leq i_{1} \leq \cdots \leq i_{m}, j_{k} \geq \cdots \geq j_{1} \geq 0, i_{m} \neq j_{k}$ and if $x_{i}$ and $x_{i}^{-1}$ appear in the decomposition of $x$, then so does $x_{i+1}$ or $x_{i+1}^{-1}$.

(3) Let $a, b$ be dyadic rational numbers with $0 \leq a<b \leq 1$ such that $b-a$ is a power of 2 . The subgroup of $F$ consisting of all functions with support in $[a, b]$ is isomorphic to $F$ where the support of $x \in F$ is $\operatorname{supp}(x)=\{t \in[0,1]: x(t) \neq t\}$.

(4) Every proper quotient group of $F$ is abelian.

(5) The commutator subgroup $F^{\prime}=[F, F]$ is normal and simple. 
The form in (2) is called the normal form of $x$. As a consequence of (4) and (5) of Theorem 2.3, we can see that any non-trivial normal subgroup of $F$ must contain $F^{\prime}$. In the geometric realization of the Thompson group $F$, the corresponding homeomorphisms $x_{n}$ are defined by

$$
x_{n}(t)= \begin{cases}t, & 0 \leq t \leq 1-\frac{1}{2^{n}} \\ \frac{t}{2}+\frac{1}{2}\left(1-\frac{1}{2^{n}}\right), & 1-\frac{1}{2^{n}} \leq t \leq 1-\frac{1}{2^{n+1}}, \\ t-\frac{1}{2^{n+2}}, & 1-\frac{1}{2^{n+1}} \leq t \leq 1-\frac{1}{2^{n+2}}, \\ 2 t-1, & 1-\frac{1}{2^{n+2}} \leq t \leq 1 .\end{cases}
$$

Moreover, we can see that the commutator subgroup $F^{\prime}$ of $F$ is the set

$$
\left\{f \in F: \exists \epsilon, \delta \in(0,1) \text { such that } f_{\mid[0, \epsilon]}=i d \text { and } f_{[[\delta, 1]}=i d\right\} .
$$

It is not difficult to see that the Thompson group $F$ is an i.c.c. group and therefore the associated von Neumann algebra $L(F)$ is a $\mathrm{II}_{1}$-factor. Jolissaint [12] showed that the Thompson group $F$ is inner amenable, in the sense that there is a mean on $l^{\infty}(F \backslash\{e\})$ which is invariant under conjugation. Indeed, if $\omega$ is a free ultra-filter on $\mathbb{N}$, then the linear functional $m$ on $l^{\infty}(F \backslash\{e\})$ given by

$$
m(f)=\lim _{n \rightarrow \omega} \frac{1}{n} \sum_{k=n+1}^{2 n} f\left(x_{k}\right)
$$

is an invariant mean under conjugation where the sequence $\left\{x_{k}\right\}$ is the generators with the relation $x_{i}^{-1} x_{n} x_{i}=x_{n+1}$ $(0 \leq i<n)$. He also proved that the Thompson group factor $L(F)$ has the McDuff property [13] and that $L\left(F^{\prime}\right)$ is inner asymptotically abelian where $F^{\prime}$ is the commutator subgroup of $F$ [14].

A separable $C^{*}$-algebra $\mathcal{A}$ is residually finite dimensional $(R F D)$ if for each non-zero $x \in \mathcal{A}$ there exists a $*$-homomorphism $\pi: \mathcal{A} \rightarrow \mathscr{B}$ such that $\operatorname{dim}(\mathcal{B})<\infty$ and $\pi(x) \neq 0$. Equivalently, $\mathcal{A}$ is embedded in a $C^{*}$-algebra of the form $\prod_{n=1}^{\infty} M_{k(n)}(\mathbb{C})$, where $M_{k}(\mathbb{C})$ is the algebra of $k \times k$ matrices over the complex numbers. Haagerup and Picioroaga [7] recently proved that both the reduced $C^{*}$-algebra $C_{r}^{*}(F)$ and the full $C^{*}$-algebra $C^{*}(F)$ associated with $F$ are not residually finite dimensional. This non-RFD of $C^{*}(F)$ is a weaker property than non-amenability of $F$.

The operator algebra analogue of von Neumann's conjecture on embeddings of non-abelian free groups into nonamenable groups is: Does any non-hyperfinite $I_{1}$-factor contain a copy of a free group factor $L\left(\mathbb{F}_{2}\right)$ on two generators? This is still open and the Thompson group $F$ is also expected to be a counterexample of this question.

\section{On some factors generated by $F$ and some operators}

As a vector in $l^{2}(G)$, each operator in $L(G)$ can be expressed as an $l^{2}$ sequence, that is, if $T \in L(G)$, then $T=$ $\sum_{g \in G} \lambda_{g} L_{g}$ with $\sum_{g \in G}\left|\lambda_{g}\right|^{2}<\infty$. The subset $\left\{g \in G: \lambda_{g} \neq 0\right\}$ of $G$ is called the support of $T$ and denoted by supp $T$. In convention, we will also denote $L_{g}$ by $g$ for each element $g \in G$. Let $G=G_{1} * \cdots * G_{n}(n \in\{2,3, \ldots\} \cup\{\infty\})$ be a free product of at least two but at most countably many cyclic groups. In [20], Szymański and Zhang proved that the weak closure $W^{*}\left(G, P_{\Lambda}\right)$ of $C^{*}\left(G, P_{\Lambda}\right)$ is a type $\mathrm{II}_{\infty}$-factor or a type $\mathrm{I}_{\infty}$-factor where $C^{*}\left(G, P_{\Lambda}\right)$ is a $C^{*}$-algebra generated by the reduced group $C^{*}$-algebra $C_{r}(G)$ and a collection $P_{\Lambda}$ of projections onto the $l^{2}$-spaces over certain subsets of $\Gamma$.

For $x \in F \backslash\{e\}$, let $F(x)$ be the set of all normal forms in $F$ whose initial segments coincide with $x$, that is, the set of all normal forms in $F$ starting with $x$. Let $P_{x}$ denote the orthogonal projection from $l^{2}(F)$ onto $l^{2}(F(x))$. Let $C^{*}\left(F, P_{x}\right)$ be the $C^{*}$-subalgebra of $\mathcal{B}\left(l^{2}(F)\right)$ generated by the reduced group $C^{*}$-algebra $C_{r}^{*}(F)$ and the projection $P_{x}$, and let $W^{*}\left(F, P_{x}\right)$ be the weak closure of $C^{*}\left(F, P_{x}\right)$ in $\mathcal{B}\left(l^{2}(F)\right)$, that is, the von Neumann algebra generated by the group von Neumann algebra $L(F)$ and $P_{x}$.

Theorem 3.1 ([9]). Let $F=\left\langle x_{0}, x_{1}, \ldots \mid x_{i}^{-1} x_{n} x_{i}=x_{n+1}, 0 \leq i<n\right\rangle$ be the Thompson group. Then we have that

$$
W^{*}\left(F, P_{x}\right) \cong \begin{cases}\mathcal{B}(\mathcal{H}) & \text { if the normal form of } x \text { contains some } x_{i}^{-1}, \\ \text { type } \mathrm{II} \text {-factor } & \text { otherwise. }\end{cases}
$$

We now turn to the transitive algebra question imposed by Kadison [15] which is closely related to the invariant subspace problem. Let $\mathcal{X}$ be a subset of a $\mathrm{II}_{1}$-factor $\mathcal{M}$. We say that $\mathcal{X}$ has a non-trivial invariant projection in $\mathcal{M}$ if there is a projection $P \in \mathcal{M}$ such that $P \neq 0, I$ and $T P=P T P$ for all $T \in \mathcal{X}$. We call a subset (or a subalgebra) $X$ of a $\mathrm{II}_{1}$-factor $\mathcal{M}$ transitive with respect to $\mathcal{M}$ if $\mathcal{X}$ has no non-trivial invariant projections in $\mathcal{M}$. Simply, we say that $\mathcal{X}$ is transitive in $\mathcal{M}$. This definition is similar to the original definition of transitivity (in the factor of type $\mathrm{I}_{\infty}$ ).

If $\mathscr{B}$ is a subalgebra (not necessarily $*$-subalgebra) of $\mathscr{B}(\mathscr{H})$ and $\mathscr{B}$ has no non-trivial common invariant subspace in the Hilbert space $\mathscr{H}$, then is $\mathcal{B}$ strong-operator dense in $\mathscr{B}(\mathscr{H})$ ? This question is well-known as the transitive algebra question [15]. Arveson [1] proved that if $\mathscr{A}$ is a transitive (not necessarily $*$ ) subalgebra of $\mathscr{B}(\mathscr{H}$ ) which contains a selfadjoint maximal abelian subalgebra, then $\mathcal{A}$ is strong-operator dense in $\mathscr{B}(\mathscr{H})$. Even though this question has been considered by many people, it is still open. See the monograph [19] for a general discussion of the transitive algebra 
question and related topics. If $\mathfrak{A}$ is a transitive (not necessarily norm-closed) algebra (algebraically) generated by selfadjoint operators on $\mathcal{H}$, then the strong-operator closure of $\mathfrak{A}$ in $\mathscr{B}(\mathscr{H})$ is a von Neumann algebra and must be equal to $\mathscr{B}(\mathscr{H})$. What is the situation if $\mathfrak{A}$ is generated by isometries or normal operators? In spite of a great deal of interest in this question, no transitive algebras other than $\mathcal{B}(\mathscr{H})$ have yet known.

In a factor of type $\mathrm{II}_{1}$, there are many non-trivial strong-operator closed transitive subalgebras. Furthermore, the transitive algebra question could also be considered for algebras generated by special kinds of operators. To find nontrivial transitive algebras, we considered some factor $\mathcal{M}$ of type $\mathrm{II}_{1}$ and some elements from its commutant $\mathcal{M}^{\prime}$. We proved that some two unitaries with the irrational rotation relation in the hyperfinite $\mathrm{II}_{1}$-factor $\mathcal{R}$ and the commutant $\mathcal{R}^{\prime}$ generate $\mathcal{B}(\mathscr{H})$ and that the algebra (algebraically) generated by only two generators of $L\left(\mathbb{F}_{\infty}\right)$ and the commutant $L\left(\mathbb{F}_{\infty}\right)^{\prime}$ is strong-operator dense in $\mathcal{B}(\mathscr{H})$, where $\mathbb{F}_{\infty}$ is the free group with countably infinite generators. See [10] for more details.

Theorem 3.2 ([9]). Let $x_{0}$ and $x_{1}$ be generators of the Thompson group $F$. Then the (non-selfadjoint) algebra generated by $\left\{L_{x_{0}}, L_{x_{1}}\right\}$ together with the commutant $L(F)^{\prime}$ is strong-operator dense in $\mathcal{B}(\mathcal{H})$ where $\mathscr{H}$ is a Hilbert space $l^{2}(F)$.

In the course of proving the above theorem one can see the following proposition.

Proposition 3.3 ([9]). Let $x_{0}$ be one of generators in $F$. We see that $L_{x_{0}^{-1}}$ is in the strong operator closure of the algebra generated by $L_{x_{0}}$ and the commutant $L(F)^{\prime}$.

The operator $L_{x_{0}}+L_{x_{1}}$ has a non-trivial invariant projection in $L(F)$. Furthermore, it is known that $L(F)$ is a McDuff factor of type $\mathrm{II}_{1}$, so that $L(F)$ is singly generated. See [9] for some detailed discussion.

\section{Amenable action and co-amenability of the Thompson group}

Let $X$ be a non-empty set. A mean on $X$ is a map $\mu: \mathcal{P}(X) \rightarrow[0,1]$ such that $\mu(X)=1$ and $\mu(A \cup B)=\mu(A)+\mu(B)$ if $A \cap B=\varnothing$.

Definition 4.1. Let a group $G$ act on a set $X$. A set $X$ admits a paradoxical decomposition if there are a partition $X_{1}, \ldots, X_{m}, Y_{1}, \ldots, Y_{n}$ of $X$ and elements $g_{1}, \ldots, g_{m}, h_{1}, \ldots, h_{n}$ of $G$ such that $\left\{g_{1} X_{1}, \ldots, g_{m} X_{m}\right\}$ is a partition of $X$ and $\left\{h_{1} Y_{1}, \ldots, h_{n} Y_{n}\right\}$ is a partition of $X$.

It is easy to see that the existence of such a decomposition for $X$ implies that there is no $G$-invariant mean on $X$. Given a group $G$ acting on a set $X$, one can ask when there is a $G$-invariant mean on $X$.

Definition 4.2. Let $X$ be a non-empty set and $G$ be a group.

(i) A group action $G \curvearrowright X$ is amenable if there is a $G$-invariant mean $\mu$ on $X$, i.e. $\mu(A)=\mu(g A)$ for all $g \in G$ and $A \subseteq X$

(ii) $G$ is amenable if the action $G \curvearrowright G$ is amenable.

We observe that if $G$ is amenable then every action of $G$ on any set is amenable. Recall that a subgroup $H<G$ is co-amenable if the $G$-action on $G / H$ is amenable. This is equivalent to the relative fixed point property that every continuous affine $G$-action on a convex subset of a locally convex space with an $H$-fixed point has a $G$-fixed point.

Proposition 4.3 ([17]). (1) If $H$ is a normal subgroup in $G$, then the co-amenability of $H<G$ is equivalent to amenability of the quotient group $G / H$.

(2) For a triple $K<H<G$ of groups co-amenability of $K$ in $H$ and of $H$ in $G$ implies that $K$ is co-amenable in $G$.

It is unknown whether the Thompson group $F$ is amenable or not. In the rest of this section, we will work on co-amenability of subgroups in $F$ for study of the amenability question of $F$.

Lemma 4.4 ([11]). Let $X$ be the set of dyadic rational numbers in $[0,1]$ and let $F^{\prime}$ be the commutator subgroup of $F$.

(1) The action $F^{\prime} \curvearrowright X$ is amenable.

(2) $F^{\prime}$ is co-amenable in $F$.

From Lemma 4.4 we can get the following theorem.

Theorem 4.5 ([11]). The action $F \curvearrowright X$ is amenable.

Let $I \subset(0,1)$ be any closed interval other than one point. We denote by $F_{I}$ the set of homeomorphisms $x:[0,1] \rightarrow$ $[0,1]$ in $F$ such that $\operatorname{supp}(x)=\{t \in[0,1]: x(t) \neq t\}$ is contained in the interval $I$.

Theorem 4.6 ([11]). The subgroup $F_{I}$ is co-amenable in $F$. 


\section{Order two automorphism on the Thompson group}

In this section we discuss an automorphism of the Thompson group factor $L(F)$ which is of order two. This is motivated by Connes' remarkable fundamental theorem. Let $\mathcal{M}$ be a factor of type $\mathrm{II}_{1}$. We denote by Aut $(\mathcal{M})$ (respectively, $\operatorname{Inn}(\mathcal{M})$ ) the automorphism (respectively, inner automorphism) group of $\mathcal{M}$ with the topology of strong pointwise convergence in $\mathcal{M}$. Let $\overline{\operatorname{Inn}(\mathcal{M})}$ be the closure of $\operatorname{Inn}(\mathcal{M})$ with respect to the strong pointwise convergence topology, which is called the approximately inner automorphism group.

Theorem 5.1 ([4]). Let $\mathcal{M}$ be a $\mathrm{II}_{1}$-factor.

(1) $\mathcal{M}$ is hyperfinite if and only if the symmetry $\alpha: \mathcal{M} \otimes \mathcal{M} \rightarrow \mathcal{M} \otimes \mathcal{M}, x \otimes y \mapsto y \otimes x$, is in $\overline{\operatorname{Inn}(\mathcal{M} \otimes \mathcal{M})}$.

(2) A discrete i.c.c. group $G$ is amenable if and only if $L(G)$ is hyperfinite.

We consider an automorphism of the Thompson group factor $L(F)$. In particular, we will consider an order two automorphism $\alpha: L(F) \rightarrow L(F)$ since a symmetry is an automorphism of order two. In order to see if $F$ is amenable or non-amenable, we investigate if $\alpha$ is approximately inner.

To get such an automorphism, we first consider the geometric realization of the Thompson group $F$. Take an order two automorphism $\alpha: F \rightarrow F$ which rotates each element in $F$ by $180^{\circ}$ with a center $\left(\frac{1}{2}, \frac{1}{2}\right)$. Then $\alpha$ is the automorphism of $F$ given by $\alpha(x)(t)=1-x(1-t)$. We still denote by the same notation $\alpha$ the automorphism on $L(F)$ induced by $\alpha$. Then we can see that

$$
\alpha\left(x_{0}\right)=x_{0}^{-1} \quad \text { and } \quad \alpha\left(x_{1}\right)=x_{0} x_{1} x_{0}^{-2} .
$$

Inductively, we can find that $\alpha\left(x_{n}\right)=x_{0}^{n} x_{1} x_{0}^{-n-1}$ for $n \geq 1$. Then such $\alpha$ extends to an automorphism of $L(F)$

Proposition 5.2. The automorphism $\alpha$ of $L(F)$ is an outer automorphism of order two.

However, we don't know whether or not $\alpha$ is approximately inner. Since the Thompson group is expected to be nonamenable by many people, we can ask if $\alpha$ is approximately inner in order to show that the Thompson group $F$ is nonamenable. If we could prove that $\alpha$ is not approximately inner, then by it follows from Theorem 5.1 that the Thompson group factor $L(F)$ will be not hyperfinite, so that $F$ will be not amenable.

\section{Acknowledgment}

The author would like to thank the referee for careful reading and various comments and suggestions. This work was supported by the Korea-Japan Basic Scientific Cooperation Program "Noncommutative Stochastic Analysis and Its Applications to Network Science."

\section{REFERENCES}

[1] Arveson, W., “A density theorem for operator algebras," Duke Math. J., 23: 635-648 (1967).

[2] Brin, M., and Squier, C., "Groups of piecewise linear homeomorphisms of the real line," Invent. Math., 79: 485-498 (1985).

[3] Cannon, J., Floyd, W., and Parry, W., "Introductory notes on Richard Thompson's groups," Enseign. Math., 42(2): 215-256 (1996).

[4] Connes, A., "Classification of injective factors, Cases $\mathrm{II}_{1}, \mathrm{II}_{\infty}, \mathrm{III}_{\lambda}, \lambda \neq 1$," Ann. of Math., 104: $73-115$ (1976).

[5] Effros, E., "Property $\Gamma$ and inner amenability," Proc. Amer. Math. Soc., 47: 483-486 (1975).

[6] Grigorchuk, R., "An example of a finitely presented amenable group that does not belong to the class EG," Mat. Sb., 189: 79-100 (1998).

[7] Haagerup, U., and Picioroaga, G., New presentations of Thompson's group and applications, to appear in J. Operator Theory.

[8] Haagerup, U., and Schultz, H., Invariant subspaces for operators in a general II $_{1}$-factor, to appear in Publ. Math. Inst. Hautes Etudes Sci.

[9] Heo, J., "On the Thompson group factor," J. Operator Theory, 53: 185-195 (2005).

[10] Heo, J., On transitive operator algebras containing the free group von Neumann algebras, preprint (2006).

[11] Heo, J., On amenable action and co-amenability of the Thompson group, in preparation.

[12] Jolissaint, P., "Moyennabilité intérieure du groupe $F$ de Thompson," C. R. Acad. Sci. Paris Sér. I Math., 325: 61-64 (1997).

[13] Jolissaint, P., "Central sequences in the factor associated with Thompson's group F," Ann. Inst. Fourier (Grenoble), 48: 10931106 (1998).

[14] Jolissaint, P., “Operator algebras related to Thompson's group F," J. Aust. Math. Soc., 79: 231-241 (2005).

[15] Kadison, R., "On the orthogonalization of operator representations," Amer. J. Math., 78: 600-621 (1955).

[16] Kadison, R., and Ringrose, J., "Fundamentals of the Theory of Operator Algebras I, II," American Mathematical Society, Providence, RI (1997).

[17] Monod, N., and Popa, S., "On co-amenability for groups and von Neumann algebras," C. R. Math. Acad. Sci. Soc. R. Can., 25: 82-87 (2003).

[18] Ol'shanskii, A., and Sapir, M., "Non-amenable finitely presented torsion-by-cyclic groups," Publ. Math. Inst. Hautes Études Sci., 96: 43-169 (2002). 
[19] Radjavi, H., and Rosenthal, P., "Invariant Subspaces," Springer-Verlag, Berlin (1973).

[20] Szymański, W., and Zhang, S., "Type $\mathrm{II}_{\infty}$ factors generated by purely infinite simple $C^{*}$-algebras associated with free groups," Proc. Amer. Math. Soc., 128: 813-818 (2000). 